Article

\title{
Staphylococcus saccharolyticus: An Overlooked Human Skin Colonizer
}

\author{
Charlotte M. Ahle 1,2®, Kristian Stødkilde ${ }^{3}$, Mastaneh Afshar ${ }^{3}$, Anja Poehlein ${ }^{4}$, \\ Lesley A. Ogilvie ${ }^{5}$, Bo Söderquist ${ }^{6}$, Jennifer Hüpeden ${ }^{1}$ and Holger Brüggemann ${ }^{3, * \mathbb{C}}$ \\ 1 Beiersdorf AG, Research \& Development, Front End Innovation, 20245 Hamburg, Germany; \\ charlotte.ahle@beiersdorf.com (C.M.A.); jennifer.huepeden@beiersdorf.com (J.H.) \\ 2 Department of Microbiology and Biotechnology, University of Hamburg, 22609 Hamburg, Germany \\ 3 Department of Biomedicine, Aarhus University, 8000 Aarhus, Denmark; kst@biomed.au.dk (K.S.); \\ m.afshar@biomed.au.dk (M.A.) \\ 4 Department of Genomic and Applied Microbiology, Institute of Microbiology and Genetics, \\ University of Göttingen, 37073 Göttingen, Germany; anja.poehlein@biologie.uni-goettingen.de \\ 5 Max Planck Institute for Molecular Genetics, 14195 Berlin, Germany; logilvie@molgen.mpg.de \\ 6 Department of Laboratory Medicine, Clinical Microbiology, Faculty of Medicine and Health, \\ Örebro University, S-701 82 Örebro, Sweden; bo.soderquist@oru.se \\ * Correspondence: brueggemann@biomed.au.dk
}

Received: 7 July 2020; Accepted: 21 July 2020; Published: 23 July 2020

\begin{abstract}
Coagulase-negative staphylococcal species constitute an important part of the human skin microbiota. In particular, facultative anaerobic species such as Staphylococcus epidermidis and Staphylococcus capitis can be found on the skin of virtually every human being. Here, we applied a culture-independent amplicon sequencing approach to identify staphylococcal species on the skin of healthy human individuals. While S. epidermidis and S. capitis were found as primary residents of back skin, surprisingly, the third most abundant member was Staphylococcus saccharolyticus, a relatively unstudied species. A search of skin metagenomic datasets detected sequences identical to the genome of S. saccharolyticus in diverse skin sites, including the back, forehead, and elbow pit. Although described as a slow-growing anaerobic species, a re-evaluation of its growth behavior showed that S. saccharolyticus can grow under oxic conditions, and, in particular, in a $\mathrm{CO}_{2}$-rich atmosphere. We argue here that $S$. saccharolyticus was largely overlooked in previous culture-dependent and -independent studies, due to its requirement for fastidious growth conditions and the lack of reference genome sequences, respectively. Future studies are needed to unravel the microbiology and host-interacting properties of $S$. saccharolyticus and its role as a prevalent skin colonizer.
\end{abstract}

Keywords: Staphylococcus saccharolyticus; coagulase-negative staphylococci; skin microbiota; skin microbiome; amplicon next generation sequencing

\section{Introduction}

In recent years, new discoveries regarding the composition and functionality of the human skin microbiota have been made, that have enabled a more comprehensive description of this ecosystem [1-3]. Studies highlighted the diversity and uniqueness of the collection of skin microorganisms with essential roles in protection against harmful pathogens, maintaining skin homeostasis, and priming our immune system [3-6].

Coagulase-negative staphylococci (CoNS) constitute an important part of the human skin microbiota. Culture-dependent and -independent studies have highlighted the ubiquity of CoNS, which colonize mostly moist and sebaceous areas of the skin. In this regard, the CoNS species Staphylococcus epidermidis, Staphylococcus capitis, and Staphylococcus hominis occupy many human skin 
sites [1,2,6-9]. Other CoNS species, such as Staphylococcus haemolyticus, Staphylococcus lugdunensis, and Staphylococcus warneri, can be found in lower amounts, varying from person to person and from skin site to skin site $[1,2,9,10]$. Some other CoNS species, such as Staphylococcus equorum, are primarily found in food products [11], but are also transient colonizers of human skin.

Culture-dependent approaches have been often applied in the past to isolate major skin residents. Such approaches are often biased, as cultivation results do not reflect the true distribution of the individual members of the microbiota [12,13]. The bias is introduced due to the chosen growth media, as well as the conditions of growth, such as $\mathrm{O}_{2}$ and $\mathrm{CO}_{2}$ concentrations, growth temperature, and cultivation time. Fast-growing microorganisms have a growth advantage, and directly or indirectly inhibit the growth of slow-growing microorganisms [6]. Therefore, culture-independent studies employing next generation sequencing (NGS)-based approaches are more frequently used in recent years. Using 16S rRNA gene amplicon-NGS, it was shown that the genus Staphylococcus is the third most abundant genus on the skin [14]. In addition, shotgun NGS studies further unraveled the diversity and individuality of staphylococcal species on the skin, and also provided insights into the strain level distribution of these CoNS species [1,2]. Such studies also highlighted the existence of microbial dark matter in the form of unidentified bacterial skin residents. For instance, the study of Oh et al. [1] has identified several uncharacterized genomes (assembled from shotgun NGS data) of unknown species, possibly belonging to the genera Corynebacterium, Cutibacterium, and Staphylococcus. Thus, it can be expected that species exist on the skin that cannot be easily cultivated by standard conditions.

In this context, we have recently described the genomes of seven strains of Staphylococcus saccharolyticus, an unusual CoNS species, regarding its growth properties [15]. Unlike almost all other CoNS species known to date, $S$. saccharolyticus largely depends on anaerobic conditions for growth, and requires fastidious growth media and prolonged cultivation time ( $>3$ days). To date, this species has been relatively uncharacterized, with limited reports on its association with implant-associated infections [15] and bacteremia [16]. Interestingly, however, culture-dependent studies have suggested that this species may also be a resident of the skin microbiota $[17,18]$.

Here, we further investigated the composition and relative abundance of staphylococcal species on human skin. For this, we applied an amplicon-NGS approach based on a Staphylococcus-specific gene fragment, to survey its presence on human back skin samples from healthy volunteers. We detected an unprecedented high relative abundance of $S$. saccharolyticus in these samples. Supported by investigation of existing skin-derived metagenomic datasets, we posit that $S$. saccharolyticus constitutes a common member of the human skin microbiota.

\section{Materials and Methods}

\subsection{Study Design and Sampling}

Skin swab samples from 19 volunteers (female, $n=11$; male, $n=8$ ) with an age range of 22-43 years were taken from the upper back. None of the volunteers had a history of skin disease; none had undergone treatment with topical medicine or antibiotics during the last six months. Written informed consent was obtained from all volunteers, and the study was approved by International Medical \& Dental Ethics Commission GmbH (IMDEC).

An area of $25 \mathrm{~cm}^{2}$ on the upper back was sampled with a cotton swab pre-moistened in aqueous sampling buffer containing disodium phosphate (12.49 g/L, Merck, Darmstadt, Germany), potassium dihydrogen phosphate (0.63 g/L, Merck, Darmstadt, Germany), and Triton X-100 (1 g/L, Merck, Darmstadt, Germany). After sampling, the swab was transferred into a sterile tube containing $2 \mathrm{~mL}$ of sampling buffer; the swab was vigorously shaken in the sampling buffer and then removed. Skin swab material was stored at $-20{ }^{\circ} \mathrm{C}$ until further processing. 


\subsection{DNA Extraction}

DNA from the $2 \mathrm{~mL}$ sample was extracted using the DNeasy PowerSoil Kit (QIAGEN, Hilden, Germany) following the manufacturer's protocol, with an additional cell lysis step using lysostaphin $(0.05 \mathrm{mg} / \mathrm{mL}$, Merck, Darmstadt, Germany) and lysozyme $(9.5 \mathrm{mg} / \mathrm{mL}$, Merck, Darmstadt, Germany) prior to extraction. DNA concentrations were measured using the Qubit dsDNA HS Assay (ThermoFisher Scientific, Waltham, MA, USA) with a Qubit fluorometer following the manufacturer's instructions.

\subsection{Amplicon PCR}

A fragment of the tuf gene present in the genomes of all staphylococcal species available in GenBank (as of December 2019) was used for species identification, analogous to a previous study using a different tuf gene fragment [19]. The target sequence was amplified using tuf-specific primers that contained MiSeq adapter sequences: tuf2_F, 5'-TCGTCGGCAGCGTCAGATGTGTAT AAGAGACAGACAGGCCGTGTTGAACGTG-3'; tuf2_R, 5' -GTCTCGTGGGCTCGGAGATGTGT ATAAGAGACAGACAGTACGTCCACCTTCACG-3'.

PCR reaction mixtures were made in a total volume of $25 \mu \mathrm{l}$ and comprised $5 \mu \mathrm{l}$ of DNA sample, $2.5 \mu \mathrm{l}$ AccuPrime PCR Buffer II (Invitrogen, Waltham, MA, USA), $1.5 \mu$ l of each primer $(10 \mu \mathrm{M})$ (DNA Technology, Risskov, Denmark), $0.15 \mu$ l AccuPrime Taq DNA Polymerase High Fidelity (Invitrogen, Waltham, MA, USA), and $14.35 \mu$ l of PCR grade water. The PCR reaction was performed using the following cycle conditions: an initial denaturation at $94^{\circ} \mathrm{C}$ for $2 \mathrm{~min}$, followed by 35 cycles of denaturation at $94^{\circ} \mathrm{C}$ for $20 \mathrm{sec}$, annealing at $55^{\circ} \mathrm{C}$ for $30 \mathrm{sec}$, elongation at $68^{\circ} \mathrm{C}$ for $1 \mathrm{~min}$, and a final elongation step at $72{ }^{\circ} \mathrm{C}$ for $5 \mathrm{~min}$. PCR products were verified on an agarose gel and purified using the Qiagen Generead ${ }^{\mathrm{TM}}$ Size Selection kit (Qiagen, Hilden, Germany). The concentration of the purified PCR products was measured with a NanoDrop 2000 spectrophotometer (ThermoFisher Scientific, Waltham, MA, USA).

\subsection{Amplicon Next Generation Sequencing}

PCR products were used to attach indices and Illumina sequencing adapters using the Nextera XT Index kit (Illumina, San Diego, CA, USA). Index PCR was performed using $5 \mu l$ of template PCR product, $2.5 \mu \mathrm{l}$ of each index primer, $12.5 \mu \mathrm{l}$ of 2x KAPA HiFi HotStart ReadyMix, and $2.5 \mu \mathrm{l}$ PCR grade water. The thermal cycling scheme was as follows: $95^{\circ} \mathrm{C}$ for $3 \mathrm{~min}, 8$ cycles of $30 \mathrm{sec}$ at $95^{\circ} \mathrm{C}, 30 \mathrm{sec}$ at $55^{\circ} \mathrm{C}$, and $30 \mathrm{sec}$ at $72{ }^{\circ} \mathrm{C}$, and a final extension at $72{ }^{\circ} \mathrm{C}$ for $5 \mathrm{~min}$. Quantification of the products was performed using the Quant-iT dsDNA HS assay kit (ThermoFisher Scientific, Waltham, MA, USA) and a Qubit fluorometer, following the manufacturer's instructions. MagSi-NGS ${ }^{\text {PREP }}$ Plus Magnetic beads (Steinbrenner Laborsysteme $\mathrm{GmbH}$, Wiesenbach, Germany) were used for purification of the indexed products as recommended by the manufacturer, and normalization was performed using the Janus Automated Workstation from Perkin Elmer (Perkin Elmer, Waltham, MA, USA). Sequencing was conducted using an Illumina MiSeq platform with dual indexing and the MiSeq reagent kit v3 (600 cycles), as recommended by the manufacturer.

\subsection{Bioinformatics}

FASTQ sequences obtained after demultiplexing the reads and trimming the primers were imported into QIIME2 (v. 2019.7) [20]. Sequences with an average quality score lower than 20 or containing unresolved nucleotides were removed from the dataset with the split_libraries_fastq.py script from QIIME. The paired-end reads were denoised and chimeras removed with DADA2 via QIIME2, and a feature table was generated [21]. These features were then clustered with VSEARCH at a threshold of $99 \%$ identity against an in-house generated tuf allele database that contained all tuf alleles from all staphylococcal genomes available in GenBank (as of December 2019). Data were normalized, and figures were prepared in $\mathrm{R}$ with the packages ggplot2 and gplots. 


\subsection{Metagenome Database Search Strategy}

The presence of sequences similar to S. saccharolyticus within available metagenomes deposited in the Sequence Read Archive (SRA) was initially assessed using the online tool at www.searchsra. org to provide a broad overview of datasets with matches. A more detailed investigation of the level of representation of $S$. saccharolyticus in existing human skin and other human-associated metagenomic datasets (identified within the initial search of the SRA) was then conducted by mapping pooled sequencing reads from metagenomic datasets against the $S$. saccharolyticus genome sequence (strain 05B0362; GenBank accession number: QHKH00000000). Sequencing reads were obtained from the SRA and processed using Geneious Prime 2020 to remove low quality reads (Trim using BBDuk; min. $50 \mathrm{bp}$ ) and duplicates (Dedupe from BBTools), with default parameters. The resulting collections of high-quality reads were mapped against the genome sequences of $S$. saccharolyticus 05B0362, Cutibacterium acnes strain ATCC 6919 (NZ_CP023676.1) and Staphylococcus epidermidis strain ATCC 14990 (NZ_CP035288.1) using the Geneious Prime 2020 map to reference tool with the following criteria: $100 \%$ identity; no gaps or mismatches; maximum ambiguity $=1$. For each metagenomic dataset mapped, the total number of reads mapped to each reference genome was normalized by the total size of the dataset, to provide reads mapped per megabase DNA.

A more targeted search strategy was also applied using an S. saccharolyticus-specific gene as a query to search metagenomic datasets derived from back skin [1]. The hya gene encoding a hyaluronate lysase was chosen (locus tag DMB78_01130 in the genome of strain 05B0362), due to its low average nucleotide identify with hya genes from other staphylococcal species. The search was performed as an SRA nucleotide BLAST. The gene was considered as being present when the coverage exceeded $40 \%$.

\subsection{S. Saccharolyticus Growth}

S. saccharolyticus (strain DVP5-16-4677) was grown on Fastidious anaerobic agar (FAA) plates (LAB M, Bury, UK) and incubated anaerobically at $37^{\circ} \mathrm{C}$ for 4 days. For liquid growth, brain-heart infusion-yeast broth supplemented with $0.05 \%$ (w/vol) cysteine (BHCY broth) was used. The following growth conditions were evaluated and performed at $37^{\circ} \mathrm{C}$ : anoxic conditions (Oxoid AnaeroGen System; ThermoFisher Scientific, Waltham, MA, USA), and oxic conditions with and without $\mathrm{CO}_{2}$-supplementation (Oxoid $\mathrm{CO}_{2}$ Gen system; ThermoFisher Scientific, Waltham, MA, USA). Optical density (OD) data at $600 \mathrm{~nm}$ was determined until the stationary growth phase.

\section{Results}

\subsection{Amplicon Sequencing of a Tuf Gene Fragment Identified Staphylococcal Species Diversity on Back Skin Samples}

Amplicon sequencing based on a tuf gene fragment was applied on swab material derived from the upper back of 19 healthy volunteers, to determine the diversity and relative abundance of staphylococcal species. In total, twelve different staphylococcal species were identified (Figure 1A and Table S1). The majority of samples contained two or more staphylococcal species, with single species found in only two samples (S. capitis and S. epidermidis, respectively). The four most abundant staphylococcal species identified were S. epidermidis (average abundance 34.0\%), S. capitis (26.6\%), S. saccharolyticus (20.5\%), and S. hominis (6.5\%) (Figure 1B). S. saccharolyticus was identified in 8 out of the 19 samples (42\%) tested and, if present, was a dominant species, comprising a minimum of $10.9 \%$ and a maximum of $90.4 \%$ of the total reads (Figure 1C). 
A

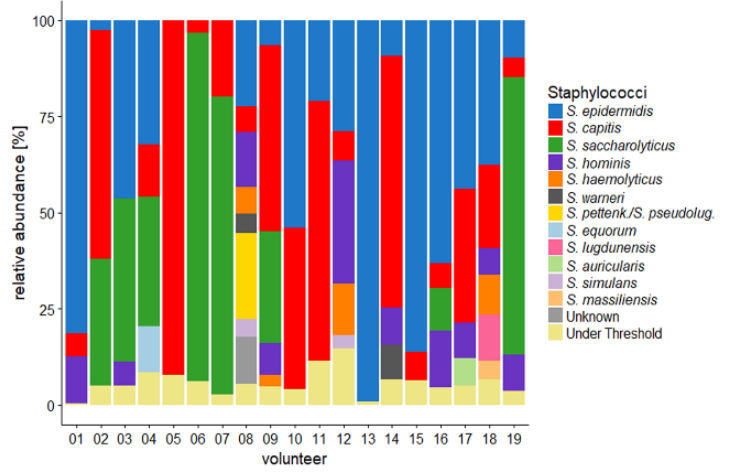

B

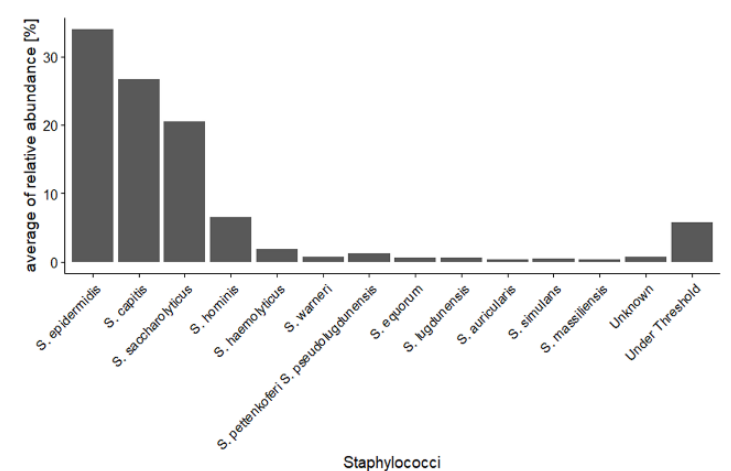

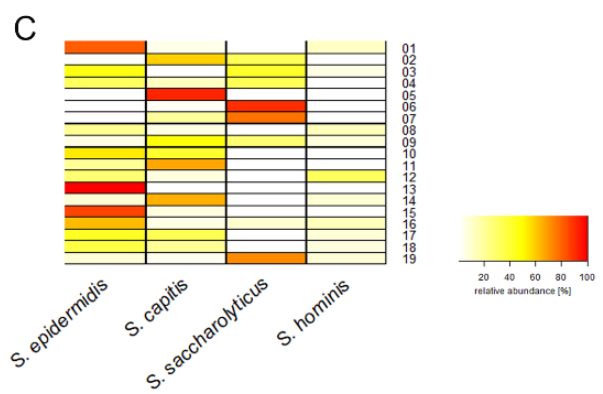

Figure 1. Diversity and abundance of staphylococcal species in back skin samples, based on amplicon next generation sequencing (NGS) data. (A) Relative abundance of staphylococcal species for each volunteer $(n=19)$. Twelve staphylococcal species were identified in the cohort using the tuf amplicon-NGS approach. The four most abundant species in the cohort were Staphylococcus epidermidis (in blue), Staphylococcus capitis (in red), Staphylococcus saccharolyticus (in green) and Staphylococcus hominis (in purple). (B) The average relative abundance of the identified 12 staphylococcal species is shown; S. epidermidis was detected with an average abundance of $34.0 \%$, S. capitis with $26.6 \%$, S. saccharolyticus with $20.5 \%$, and S. hominis with $6.5 \%$. (C) The relative abundance of the four most prevalent staphylococcal species is shown for each back skin sample in a heat map. S. epidermidis was detected in four samples with a very high abundance ( $>60 \%$ of all reads); S. capitis and S. saccharolyticus were detected with such a high abundance in three samples each.

\subsection{Presence of S. Saccharolyticus in Previous Metagenome Studies}

Given that $S$. saccharolyticus was detected in 8 out of 19 back skin samples tested, we next decided to determine the prevalence and distribution of sequences with similarity to $S$. saccharolyticus within existing skin metagenomic datasets, using two different search strategies. First, sequencing reads from all skin metagenomes derived from back, forehead, and armpit samples $(n=43)[1,2]$, available within the Sequence Read Archive (SRA), were mapped to the S. saccharolyticus genome with high stringency (100\% identity; no gaps or mismatches; maximum ambiguity $=1)$. As a comparison, we also mapped the same reads to the genomes of two bacterial species known to be abundant on human skin, Cutibacterium acnes and S. epidermidis. Reads mapping to the S. saccharolyticus genome were found in all 43 skin metagenomic datasets searched (Figure 2). The percentage of reads mapping to $C$. acnes was significantly higher for all datasets searched, as compared to S. saccharolyticus and S. epidermidis. To compare relative abundance profiles, we also conducted a survey of a limited set of other human-associated metagenomic datasets (originating from the human gut, tongue dorsum, and supragingival plaque) identified as carrying sequences with similarity to $S$. saccharolyticus, using the SRA metagenomic search tool. Sequencing reads from these human-associated metagenomic datasets were also found to map to all bacterial genomes assessed, but at magnitudes of order lower levels than observed for human skin datasets. 


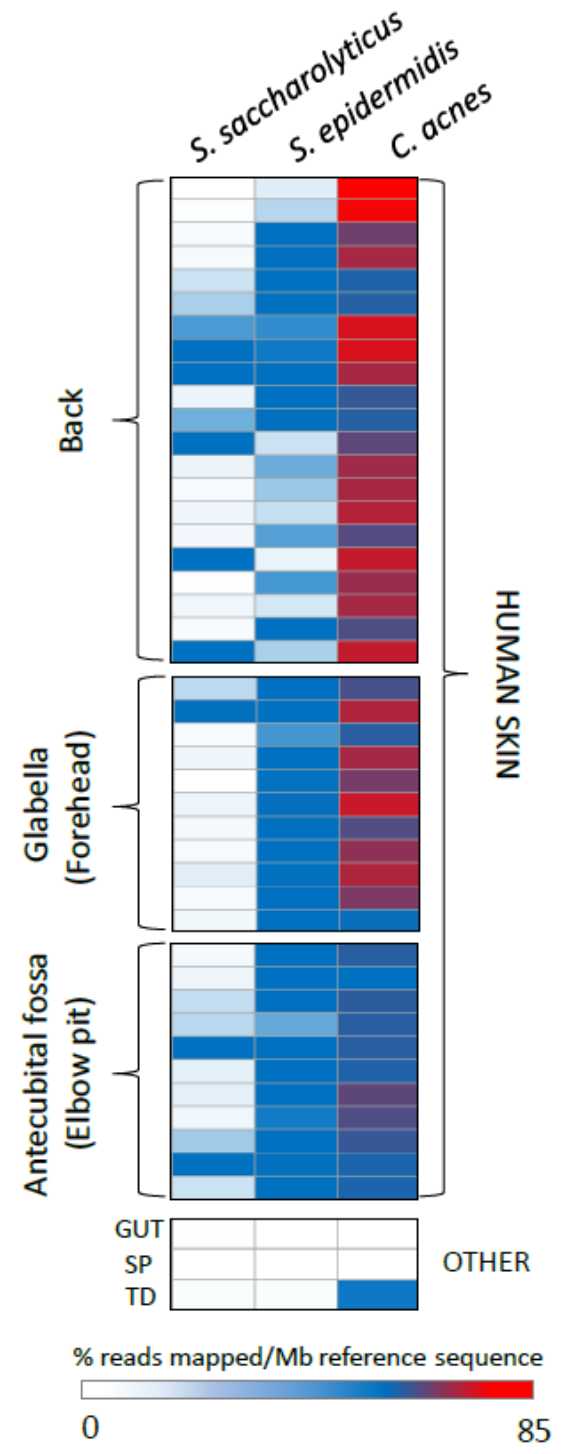

Figure 2. Representation of S. saccharolyticus in human skin and human-associated metagenomes. Heat map showing relative representation of S. saccharolyticus in metagenomes from human skin and other human associated environments. Reads from each reference metagenome were mapped to the genome sequences of $S$. saccharolyticus, S. epidermidis, and Cutibacterium acnes using high stringency criteria (100\% identity; no gaps; max. ambiguity 1$)$. The number of reads mapped was normalized for size of reference datasets (expressed as \% of reads mapped/Mb reference sequence). OTHER: non-human skin metagenomes; GUT: human gut; SP: supragingival plaque; TD: tongue dorsum. Details of the metagenomes utilized are provided in Table S3.

To complement the read mapping analyses, we also conducted a more targeted search of the shotgun NGS data derived from back skin samples of Oh el al., 2014 [1] and Oh et al., 2016 [2] (12 volunteers; samples taken at three time points per person), using the hyaluronate lyase gene of S. saccharolyticus. Using this approach, we found high-stringency matches (reads with 100\% identity) within three of the twelve volunteers at multiple time points (Table S2).

\subsection{Re-Evaluation of Growth Conditions for S. Saccharolyticus}

Our data indicate that $S$. saccharolyticus is widespread on human skin, can be detected within diverse skin sites, and may even be more abundant than S. epidermidis in some people. However, only a few studies have previously reported the presence of $S$. saccharolyticus on human skin. One possible 
reason for the previous lack of recognition is the difficulty of growing S. saccharolyticus under standard conditions. The organism cannot be detected on blood agar when incubated under aerobic conditions for 24 to $96 \mathrm{~h}$ (data not shown). Instead, it grows on Trypticase soy yeast (TSY) agar plates supplemented with $0.5 \%$ Tween-80 or FAA plates when incubated for 72 to $96 \mathrm{~h}$ under anaerobic conditions (data not shown). Not much is known about its growth in broth. Thus, we recorded the growth of $S$. saccharolyticus in brain-heart infusion-yeast broth supplemented with $0.05 \%$ cysteine (BHCY medium). Different incubation conditions were applied, including anaerobic and aerobic conditions and the supplementation with $\mathrm{CO}_{2}$ (Figure 3). Results showed that the organism grew almost equally as well under $\mathrm{CO}_{2}$-enriched conditions (approx. $6 \% \mathrm{CO}_{2}$ and $15 \% \mathrm{O}_{2}$ ), compared to anaerobic conditions. Growth yields were reduced under atmospheric conditions.

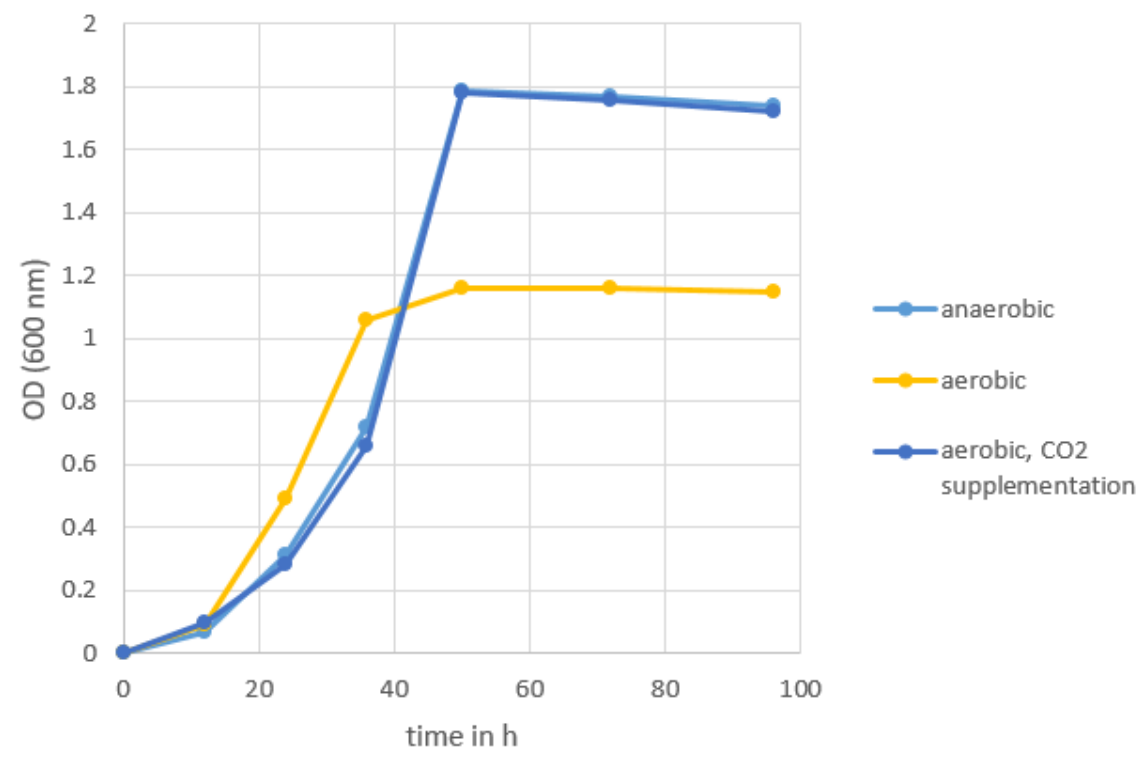

Figure 3. Growth of S. saccharolyticus in BHCY medium under different conditions. Light blue, anaerobic atmosphere (Oxoid-AnaeroGen system); dark blue, $\mathrm{CO}_{2}$-rich atmosphere (Oxoid- $\mathrm{CO}_{2}$ Gen system; generating ca. $6 \% \mathrm{CO}_{2}$, ca. $15 \% \mathrm{O}_{2}$ ); yellow, aerobic atmosphere. The experiment was replicated twice.

\section{Discussion}

The skin microbiome affects the health-state of our skin. Understanding the bacterial composition on non-diseased skin is therefore of importance. Here, we focused on the staphylococcal composition of the human back skin. Similar to most previous studies [1,2], S. epidermidis was found to be the most abundant staphylococcal species on human back skin, followed by S. capitis. Surprisingly, S. saccharolyticus ranked third. This species has previously not been reported to be abundant on human skin. However, two studies from 1978 reported the presence of S. saccharolyticus, formerly named Peptococcus saccharolyticus, in forehead and armpit skin samples $[17,18]$. In these studies, around $20 \%$ of samples were found to be positive for S. saccharolyticus. The organism grew on TSY (supplemented with $0.5 \%$ Tween-80) agar plates after 4-7 days of incubation, with preference for anaerobic conditions. Identification (and differentiation from other CoNS) was based on cell and colony morphology, anaerobic growth preference, and a weak catalase activity. Biochemically, S. saccharolyticus cannot produce lactic acid from glucose (in contrast to other staphylococci); it can ferment glucose, fructose, and glycerol, but not maltose. Interestingly, Evans et al. [17] stated that it was puzzling that the organism was not recognized in past studies "in view of its prevalence". The authors also suggested that the reason that previous skin studies have overlooked this organism was due to (i) the choice of the culture media, (ii) the need for prolonged incubation time, (iii) the preference for anaerobic culture conditions, and (iv) misidentification. Indeed, 40 years later, not much has changed in this regard. Most culture-dependent skin microbiota studies do overlook this microorganism, possibly due 
to inappropriate growth and cultivation conditions, as outlined by Evans et al. [17,18]. In addition, fast-growing species such as S. epidermidis might outcompete S. saccharolyticus on (standard) agar plates. This could explain why $S$. saccharolyticus was overlooked in culture-dependent studies.

However, this does not explain why S. saccharolyticus was previously not detected in cultureindependent studies, which are nowadays more frequently conducted. Many culture-independent studies are carried out using 16S rRNA gene amplicon sequencing, which relies on sufficient differences in the $16 \mathrm{~S}$ rRNA gene to distinguish species. However, the $16 \mathrm{~S}$ rRNA gene of $S$. saccharolyticus does not carry many single nucleotide polymorphisms (SNPs) that can easily distinguish it from other CoNS, namely $S$. capitis [15]. Thus, depending on the $16 \mathrm{~S}$ rRNA gene amplification strategy (amplifying the $\mathrm{V} 1, \mathrm{~V} 2, \mathrm{~V} 3, \mathrm{~V} 4, \mathrm{~V} 5$, and the V6 region, respectively, or a combination thereof), S. saccharolyticus can be indistinguishable from $S$. capitis.

In recent years, shotgun sequencing was employed to identify the skin metagenome $[1,2]$. However, meaningful analyses of shotgun sequencing data rely on reference genomes of all skin microorganisms. Regarding S. saccharolyticus, no such reference genome was available before March 2019. Adding to the confusion, three genomes assigned to S. saccharolyticus were publicly available in GenBank before 2019, but these were wrongly classified as S. saccharolyticus, and actually belong to S. capitis, as previously noted [15]. They were recently correctly reassigned to $S$. capitis. Besides the genomes of seven $S$. saccharolyticus strains that have been sequenced during our previous study [15], the type strain of S. saccharolyticus (ATCC 14953/NCTC 11807) has been sequenced by two independent teams (WGS projects UHDZ01 and RXWW01), resulting in nearly identical genome sequences. Taken together, before March 2019 there was no correct reference genome sequence of S. saccharolyticus available; this has been now resolved. Thus, current and future shotgun sequencing studies should be able to identify S. saccharolyticus correctly.

Here, we employed an amplicon sequencing method that is a modification from an existing method by Strube et al. [19]. The method is based on the amplification of a tuf gene fragment, with primers that were designed by Martineau et al. [22]. The tuf gene, encoding the elongation factor Tu, is a highly conserved gene in all staphylococcal species. We modified this method by choosing different amplification primers, since we noticed that the reverse primer designed by Martineau et al. [22] has two mismatches with the corresponding tuf gene sequence in the genome of $S$. saccharolyticus. It is thus likely that the primers of Martineau et al. [22] do not amplify the tuf gene of S. saccharolyticus, or only with reduced efficiency.

Previous studies reported that $S$. saccharolyticus has a preference for anaerobic growth conditions $[17,18]$. Here, we showed that the bacterium can also grow under atmospheric conditions in broth, but with a reduced growth yield compared to anaerobic conditions. However, the growth in a $\mathrm{CO}_{2}$-rich atmosphere is comparable to the growth under anaerobic conditions. A mechanistic explanation of the effect of $\mathrm{CO}_{2}$ on bacterial growth was recently published by Fan et al. [23]. The authors investigated the growth-promoting effect of $\mathrm{CO}_{2}$ and the $\mathrm{CO}_{2}$-dependency of small colony variants of $S$. aureus, whose growth defect can be compensated by increased $\mathrm{CO}_{2}$ /bicarbonate supplementation. They found that staphylococci employ a $\mathrm{CO}_{2}$-concentrating mechanism that enables them to grow at atmospheric $\mathrm{CO}_{2}$ levels. More specifically, they found that the system MpsAB is crucial for $S$. aureus growth at atmospheric $\mathrm{CO}_{2}$ levels. From a set of carefully designed experiments, they concluded that the MpsAB system represents a dissolved inorganic carbon transporter, or bicarbonate concentrating system, which creates an elevated concentration of intracellular bicarbonate. Consequently, a staphylococcal species without a functional MpsAB system would only grow poorly at atmospheric $\mathrm{CO}_{2}$ levels, and would rely on increased $\mathrm{CO}_{2}$ concentrations for accelerated growth. As we previously noted that the genome of $S$. saccharolyticus contains over 300 frameshift mutations [15], we checked the mps $A B$ genes. Indeed, the mps $A B$ genes in the genome of $S$. saccharolyticus are frameshifted, and thus the MpsAB system is most likely non-functional in S. saccharolyticus. In conclusion, the lack of a functional $\mathrm{CO}_{2}$-concentrating system in $\mathrm{S}$. saccharolyticus is a likely explanation for its insufficient growth under atmospheric conditions. This can be compensated by providing increased $\mathrm{CO}_{2}$ concentrations. 
Many questions remain, e.g., regarding the preferred niche of S. saccharolyticus on human skin and its evolutionary history that can be regarded as an example of reductive evolution, indicative of the massive genome decay [15]. Genomic modifications such as genome decay are often a result of a relative recent lifestyle change, e.g., the adaptation to a new host or a new niche within a host, associated with a strict(er) host dependency [24-26]. What could a scenario for the evolutionary history of S. saccharolyticus look like? In this regard, an interesting feature of S. saccharolyticus is the presence of a hyaluronate lyase (Hya), which is absent in other human-associated skin-resident CoNS. Closest homologs of the hya gene of S. saccharolyticus are present in two animal-associated staphylococci: Staphylococcus agnetis and Staphylococcus hyicus. S. agnetis is associated with lameness in broiler chickens, and S. hyicus causes skin diseases, such as exudative dermatitis in piglets [27,28]. It is tempting to suggest that the (horizontal) acquisition of hya, possibly from an animal-associated staphylococcal species, contributed to a lifestyle switch of $S$. saccharolyticus. Hyaluronate lyases degrade hyaluronic acid, a major polysaccharide of the extracellular matrix of tissues [29]. In the epidermis, hyaluronic acid is found in high concentrations, in particular in deeper layers of the epidermis, such as the stratum spinosum [30]. Thus, a hyaluronidase-producing $S$. saccharolyticus is likely better equipped to penetrate and propagate in deeper layers of the epidermis. We further speculate that a strong host association in deeper layers might have been established, where S. saccharolyticus would have access to a range of host-derived compounds including amino acids and cofactors. This in turn would render bacterial genes to synthesize such compounds dispensable. As a consequence, genome decay would be accelerated, aiming at a slimmer, less energy-consuming lifestyle that is adapted to an oxygen-depleted niche, i.e., the epidermis below the stratum corneum.

Another open question remains regarding the clinical significance of these findings. At present, only few studies, mainly case reports, have reported the involvement of $S$. saccharolyticus in human disease. The microorganism has been described as the etiologic agent of infective endocarditis, empyema and bone and joint infections such as shoulder synovitis and vertebral osteomyelitis [31-36]. In addition, the bacterium was reported to be responsible for nosocomial bloodstream infections in a German hospital [16]. A few reports have found S. saccharolyticus in implant-associated infections, such as prosthetic valve endocarditis [37] and we recently described eight cases of prosthetic joint infections where S. saccharolyticus was identified from tissue biopsies [15]. If S. saccharolyticus is widespread on human skin, as our study results suggest, one would expect to see more reports regarding the potential disease association of this bacterium, as seen for example for other skin-resident CoNS, such as S. epidermidis and S. capitis. As outlined above in detail, we hypothesize that mainly due to the fastidious growth conditions, S. saccharolyticus was overlooked in numerous disease cases, in particular in implant-associated infections, as such infections are often caused by skin-derived bacteria, including CoNS. In several cases, S. saccharolyticus has been identified, but was labeled as contaminant [38]. As also true for other CoNS, assigning an etiological role to S. saccharolyticus in disease requires thorough investigations to exclude skin-derived contamination of the biopsy material or contamination during subsequent sample processing steps. Carefully designed and executed future studies are needed to elucidate the etiology and frequency of $S$. saccharolyticus in human disease.

The study has some limitations, most importantly the small sample size $(n=19)$ and the focus on back skin. Moreover, only relatively young participants were investigated in this study. Appropriate skin sampling methods have previously been discussed [39]; due to the here applied sampling method, i.e., skin swabs, we harvested mainly the microbiome of the stratum corneum. Thus, microorganisms that potentially penetrate deeper skin layers of the epidermis might be underrepresented. In future studies, we aim at analyzing more individuals, thereby covering and comparing different age groups and diverse skin health conditions. In addition, different skin sites will be investigated, and different skin sampling methods applied, in order to determine the specific skin tissue location of S. saccharolyticus.

In conclusion, here we found that the coagulase-negative species $S$. saccharolyticus is relatively often found in human skin samples, as judged from a culture-independent amplicon sequencing approach. When present, the organism can comprise a major part of the staphylococcal skin population, 
and is found in several different skin sites. It has yet to be shown in the future if skin that is primarily colonized with S. saccharolyticus has distinguishable features from skin colonized with S. epidermidis or S. capitis.

Supplementary Materials: The following are available online at http:/www.mdpi.com/2076-2607/8/8/1105/s1, Table S1: Sequence read count matching the tuf gene fragment of different staphylococcal species; Table S2: Identification of sequencing reads in back skin metagenomic data that map to the hya gene of S. saccharolyticus with $100 \%$ nucleotide identity; Table S3: List of metagenomic datasets used for read mapping.

Author Contributions: Conceptualization: C.M.A., J.H., and H.B.; methodology and experiments: C.M.A., M.A., A.P., and L.A.O.; resources: B.S.; data analysis: C.M.A., K.S., A.P., L.A.O., and H.B.; writing-original draft preparation: C.M.A. and H.B.; writing-review and editing: C.M.A., K.S., M.A., A.P., L.A.O., B.S., J.H., and H.B.; supervision: J.H. and H.B.; project administration: J.H. and H.B.; funding acquisition: H.B. All authors have read and agreed to the published version of the manuscript.

Funding: This research was funded by NovoNordisk, grant number NNF18OC0053172.

Acknowledgments: We thank Lise Hald Schultz for excellent technical assistance. Data processing was performed on the GenomeDK cluster; we would like to thank GenomeDK and Aarhus University for providing computational resources that contributed to these research results.

Conflicts of Interest: C.M.A. and J.H. are employees at Beiersdorf AG. The other authors declare no conflicts of interest. The funders had no role in the design of the study; in the collection, analyses, or interpretation of data; in the writing of the manuscript; or in the decision to publish the results.

\section{References}

1. Oh, J.; Byrd, A.L.; Deming, C.; Conlan, S.; Program, N.C.S.; Kong, H.H.; Segre, J.A. Biogeography and individuality shape function in the human skin metagenome. Nature 2014, 514, 59-64. [CrossRef] [PubMed]

2. Oh, J.; Byrd, A.L.; Park, M.; Program, N.C.S.; Kong, H.H.; Segre, J.A. Temporal Stability of the Human Skin Microbiome. Cell 2016, 165, 854-866. [CrossRef] [PubMed]

3. Byrd, A.L.; Belkaid, Y.; Segre, J.A. The human skin microbiome. Nat. Rev. Microbiol. 2018, 16, $143-155$. [CrossRef] [PubMed]

4. Belkaid, Y.; Segre, J.A. Dialogue between skin microbiota and immunity. Science 2014, 346, 954-959. [CrossRef]

5. Sanford, J.A.; Gallo, R.L. Functions of the skin microbiota in health and disease. Semin. Immunol. 2013, 25, 370-377. [CrossRef]

6. Otto, M. Staphylococci in the human microbiome: The role of host and interbacterial interactions. Curr. Opin. Microbiol. 2020, 53, 71-77. [CrossRef]

7. Kloos, W.E.; Musselwhite, M.S. Distribution and persistence of Staphylococcus and Micrococcus species and other aerobic bacteria on human skin. Appl. Microbiol. 1975, 30, 381-385. [CrossRef]

8. Schoenfelder, S.M.; Lange, C.; Eckart, M.; Hennig, S.; Kozytska, S.; Ziebuhr, W. Success through diversity—how Staphylococcus epidermidis establishes as a nosocomial pathogen. Int. J. Med. Microbiol. 2010, 300, 380-386. [CrossRef]

9. Heilmann, C.; Ziebuhr, W.; Becker, K. Are coagulase-negative staphylococci virulent? Clin. Microbiol. Infect. 2019, 25, 1071-1080. [CrossRef]

10. Bieber, L.; Kahlmeter, G. Staphylococcus lugdunensis in several niches of the normal skin flora. Clin. Microbiol. Infect. 2010, 16, 385-388. [CrossRef]

11. Meugnier, H.; Bes, M.; Vernozy-Rozand, C.; Mazuy, C.; Brun, Y.; Freney, J.; Fleurette, J. Identification and ribotyping of Staphylococcus xylosus and Staphylococcus equorum strains isolated from goat milk and cheese. Int. J. Food Microbiol. 1996, 31, 325-331. [CrossRef]

12. Ward, D.M.; Weller, R.; Bateson, M.M. 16S rRNA sequences reveal numerous uncultured microorganisms in a natural community. Nature 1990, 345, 63-65. [CrossRef] [PubMed]

13. Dickson, R.P.; Erb-Downward, J.R.; Prescott, H.C.; Martinez, F.J.; Curtis, J.L.; Lama, V.N.; Huffnagle, G.B. Analysis of culture-dependent versus culture-independent techniques for identification of bacteria in clinically obtained bronchoalveolar lavage fluid. J. Clin. Microbiol. 2014, 52, 3605-3613. [CrossRef]

14. Grice, E.A.; Kong, H.H.; Conlan, S.; Deming, C.B.; Davis, J.; Young, A.C.; Program, N.C.S.; Bouffard, G.G.; Blakesley, R.W.; Murray, P.R.; et al. Topographical and temporal diversity of the human skin microbiome. Science 2009, 324, 1190-1192. [CrossRef] [PubMed] 
15. Brüggemann, H.; Poehlein, A.; Brzuszkiewicz, E.; Scavenius, C.; Enghild, J.J.; Al-Zeer, M.A.; Brinkmann, V.; Jensen, A.; Söderquist, B. Staphylococcus saccharolyticus Isolated from Blood Cultures and Prosthetic Joint Infections Exhibits Excessive Genome Decay. Front. Microbiol. 2019, 10, 478. [CrossRef] [PubMed]

16. Steinbrueckner, B.; Singh, S.; Freney, J.; Kuhnert, P.; Pelz, K.; Aufenanger, J. Facing a mysterious hospital outbreak of bacteraemia due to Staphylococcus saccharolyticus. J. Hosp. Infect. 2001, 49, 305-307. [CrossRef]

17. Evans, C.A.; Mattern, K.L.; Hallam, S.L. Isolation and identification of Peptococcus saccharolyticus from human skin. J. Clin. Microbiol. 1978, 7, 261-264. [PubMed]

18. Evans, C.A.; Mattern, K.L. Individual differences in the bacterial flora of the skin of the forehead: Peptococcus saccharolyticus. J. Investig. Dermatol. 1978, 71, 152-153. [CrossRef]

19. Strube, M.L.; Hansen, J.E.; Rasmussen, S.; Pedersen, K. A detailed investigation of the porcine skin and nose microbiome using universal and Staphylococcus specific primers. Sci. Rep. 2018, 8, 12751. [CrossRef]

20. Bolyen, E.; Rideout, J.R.; Dillon, M.R.; Bokulich, N.A.; Abnet, C.C.; Al-Ghalith, G.A.; Alexander, H.; Alm, E.J.; Arumugam, M.; Asnicar, F.; et al. Reproducible, interactive, scalable and extensible microbiome data science using QIIME 2. Nat. Biotechnol. 2019, 37, 852-857. [CrossRef]

21. Callahan, B.J.; McMurdie, P.J.; Rosen, M.J.; Han, A.W.; Johnson, A.J.; Holmes, S.P. DADA2: High-resolution sample inference from Illumina amplicon data. Nat. Methods 2016, 13, 581-583. [CrossRef] [PubMed]

22. Martineau, F.; Picard, F.J.; Ke, D.; Paradis, S.; Roy, P.H.; Ouellette, M.; Bergeron, M.G. Development of a PCR assay for identification of staphylococci at genus and species levels. J. Clin. Microbiol. 2001, 39, 2541-2547. [CrossRef] [PubMed]

23. Fan, S.H.; Ebner, P.; Reichert, S.; Hertlein, T.; Zabel, S.; Lankapalli, A.K.; Nieselt, K.; Ohlsen, K.; Gotz, F. MpsAB is important for Staphylococcus aureus virulence and growth at atmospheric $\mathrm{CO}_{2}$ levels. Nat. Commun. 2019, 10, 3627. [CrossRef] [PubMed]

24. Pallen, M.J.; Wren, B.W. Bacterial pathogenomics. Nature 2007, 449, 835-842. [CrossRef]

25. Argemi, X.; Hansmann, Y.; Prola, K.; Prévost, G. Coagulase-Negative Staphylococci Pathogenomics. Int. J. Mol. Sci. 2019, 20, 1215. [CrossRef]

26. Lindsay, J.A. Staphylococci: Evolving Genomes. Microbiol. Spectr. 2019, 7. [CrossRef]

27. Hazarika, R.A.; Mahanta, P.N.; Dutta, G.N.; Devriese, L.A. Cutaneous infection associated with Staphylococcus hyicus in cattle. Res. Vet. Sci. 1991, 50, 374-375. [CrossRef]

28. Foster, A.P. Staphylococcal skin disease in livestock. Vet. Dermatol. 2012, 23, 342-351, e63. [CrossRef]

29. Hynes, W.L.; Walton, S.L. Hyaluronidases of Gram-positive bacteria. FEMS Microbiol. Lett. 2000, 183, $201-207$. [CrossRef]

30. Tammi, R.; Ripellino, J.A.; Margolis, R.U.; Tammi, M. Localization of epidermal hyaluronic acid using the hyaluronate binding region of cartilage proteoglycan as a specific probe. J. Investig. Dermatol. 1988, 90, 412-414. [CrossRef]

31. Westblom, T.U.; Gorse, G.J.; Milligan, T.W.; Schindzielorz, A.H. Anaerobic endocarditis caused by Staphylococcus saccharolyticus. J. Clin. Microbiol. 1990, 28, 2818-2819. [CrossRef] [PubMed]

32. Godreuil, S.; Jean-Pierre, H.; Morel, J.; Darbas, H.; Jumas-Bilak, E.; Bañuls, A.L.; Marchandin, H. Unusual case of spondylodiscitis due to Staphylococcus saccharolyticus. Joint Bone Spine 2005, 72, 91-93. [CrossRef] [PubMed]

33. Mikhael, M.M.; Bach, H.G.; Huddleston, P.M.; Maus, T.P.; Berbari, E.F. Multilevel diskitis and vertebral osteomyelitis after diskography. Orthopedics 2009, 32, 60. [CrossRef] [PubMed]

34. Schneeberger, A.G.; Yian, E.; Steens, W. Injection-induced low-grade infection of the shoulder joint: Preliminary results. Arch. Orthop. Trauma Surg. 2012, 132, 1387-1392. [CrossRef]

35. Oberbach, A.; Friedrich, M.; Lehmann, S.; Schlichting, N.; Kullnick, Y.; Gräber, S.; Buschmann, T.; Hagl, C.; Bagaev, E.; CardiOmics group; et al. Bacterial infiltration in structural heart valve disease. J. Thorac. Cardiovasc. Surg. 2019, S0022-5223, 30451-30459. [CrossRef]

36. Wang, P.; Liu, Y.; Xu, Y.; Xu, Z. Staphylococcus saccharolyticus infection: Case series with a PRISMA-compliant systemic review. Med. Baltim. 2020, 99, e20686. [CrossRef]

37. Krishnan, S.; Haglund, L.; Ashfaq, A.; Leist, P.; Roat, T. Prosthetic valve endocarditis due to Staphylococcus saccharolyticus. Clin. Infect. Dis. 1996, 22, 722-723. [CrossRef] 
38. Hitzenbichler, F.; Simon, M.; Salzberger, B.; Hanses, F. Clinical significance of coagulase-negative staphylococci other than S. epidermidis blood stream isolates at a tertiary care hospital. Infection 2017, 45, 179-186. [CrossRef]

39. Omer, H.; McDowell, A.; Alexeyev, O.A. Understanding the role of Propionibacterium acnes in acne vulgaris: The critical importance of skin sampling methodologies. Clin. Dermatol. 2017, 35, 118-129. [CrossRef]

(C) 2020 by the authors. Licensee MDPI, Basel, Switzerland. This article is an open access article distributed under the terms and conditions of the Creative Commons Attribution (CC BY) license (http://creativecommons.org/licenses/by/4.0/). 